\title{
Racial/Ethnic Differences in Diet Quality and Eating Habits Among WIC Pregnant Women: Implications for Policy and Practice
}

\author{
Alla M. Hill, BS ${ }^{\circledR}$, Danielle L. Nunnery, PhD, RD², Alice Ammerman, DrPH ${ }^{3}$, \\ and Jigna M. Dharod, PhD'
}

\begin{abstract}
Purpose: One of the major federal food assistance programs, the Special Supplemental Program for Women, Infants, and Children (WIC), serves approximately I.5 million low-income pregnant women per year; however, limited information is available on their dietary habits. This is critical because low-income women are at higher risk of gaining excess weight during pregnancy. Thus, the study objectives were to (I) determine the overall diet quality of WIC pregnant women and (2) examine diet quality and eating behaviors by race/ethnicity and other sociodemographics.
\end{abstract}

Design: This was a cross-sectional study.

Setting: One of the 3 WIC offices in a north-central county in North Carolina, USA.

Sample: Pregnant women $(\mathrm{n}=198)$ in the second trimester.

Measures: Interviews included sociodemographics, food security, diet, and eating behaviors. Diet quality was assessed by the Healthy Eating Index (HEl) 2010 scores.

Analysis: Descriptives, bivariate analysis, and multivariate analysis.

Results: Average participant age was 26 years, and the mean HEl-20I0 score was 56 of maximum score of 100 . Specifically, African American women consumed significantly lower servings of whole grains $(\beta=-1.71 ; 95 \% \mathrm{Cl}:-3.10$ to $-0.32 ; P<.05)$ and dairy $(\beta=-1.42 ; 95 \% \mathrm{Cl}:-2.5 \mathrm{I}$ to $-0.33 ; P<.05)$ compared with non-Hispanic white women. Hispanic women scored higher in daily intake of fruits $(\beta=0.98 ; 95 \% \mathrm{Cl}$ : $0.17-1.79 ; P<.05)$ and for consuming empty calories in moderation $(\beta=1.57 ; 95 \% \mathrm{Cl}$ : 0.06-3.09; $P<.05)$. Frequency of intake of fast foods/outside meals was higher among African American women (57\%, $P=.025)$.

Conclusion: Efforts are warranted to promote optimal nutrition among WIC pregnant women. Specifically, African American women are highly vulnerable to poor dietary habits during pregnancy. Further investigation of barriers/facilitators for healthy eating is necessary to address nutrition disparities among WIC pregnant women.

\section{Keywords}

maternal nutrition, diet quality, nutrition disparities, pregnancy

\section{Purpose}

Eating a healthy diet is important for all life stages, but it becomes crucial during pregnancy. Poor diet quality during pregnancy is associated with gestational diabetes, dyslipidemia, preeclampsia, and other pregnancy complications. ${ }^{1-10}$ Moreover, by affecting gestational weight gain, poor diet during pregnancy has been associated with macrosomia among infants and increased risk for obesity later in childhood. ${ }^{11-13}$ In general, low-income women are more likely to have poor dietary habits than their high-income counterparts. However, the evidence on diet quality of low-income pregnant women in the United States is very limited. Among the few studies, it is seen that women living below the Federal Poverty Level (FPL)

\footnotetext{
I Department of Nutrition, The University of North Carolina at Greensboro, Greensboro, NC, USA

${ }^{2}$ Department of Nutrition and Health Care Management, Appalachian State University, Boone, NC, USA

${ }^{3}$ Department of Nutrition, The University of North Carolina at Chapel Hill, Chapel Hill, NC, USA
}

\section{Corresponding Author:}

Jigna M. Dharod, Department of Nutrition, University of North Carolina at Greensboro, 319 College Avenue, 312 Stone Building, Greensboro, NC 274I2, USA.

Email: jmdharod@uncg.edu 
consume a poor-quality diet and are at a higher risk of gaining excess weight during pregnancy. For instance, in a secondary analyses study, it was found that low-income pregnant women $(n=668)$ were meeting just $52 \%$ of the daily dietary recommendations for pregnancy. ${ }^{14}$ Further, in another study of mainly lowto middle-income pregnant women, it was found that African American women had a higher calorie intake, but a lower intake of fiber, folate, and iron compared to white women. ${ }^{15}$

The current literature indicates that cooking skills, time availability, and taste preferences are key factors affecting food choices and diet quality among low-income families. ${ }^{16,17}$ It is also seen that low-income households are more likely to experience food insecurity, which in turn is shown to negatively affect diet quality. ${ }^{18}$ Specifically, it is noted that low-income households rely more on cheaper and highly satiating and palatable foods, which are generally also high in calories, added sugars, and saturated fats.

Created specifically to address food insecurity and promote optimal nutrition during the critical life stages of pregnancy, breastfeeding, and early childhood, the Special Supplemental Program for Women, Infants, and Children (WIC) provides economic assistance to purchase specific nutrient-rich foods for low-income women and children. By income criteria, women and children living at or below $\leq 185 \%$ of FPL are eligible to participate in the WIC program. In 2018, 6.87 million people received benefit each month, of which, 1.63 million were women. ${ }^{19}$ Hence, the WIC program is vital for reaching and promoting optimal nutrition among low-income pregnant women. However, to our knowledge, very limited literature exists on diet quality and eating behaviors of WIC pregnant women. Conducting research to fill this gap is critical because excess gestational weight gain and related poor pregnancy outcomes are more common among low-income women. A clear understanding on which dietary components are of major concern and which subgroup of low-income or WIC pregnant women is highly affected by poor diet quality will help to prevent health disparities in the United States. Thus, the objectives of the study are to (1) determine overall diet quality of WIC pregnant women and (2) examine diet quality and eating behaviors by race/ethnicity and other sociodemographics.

\section{Methods}

\section{Design}

The institutional review boards of the University of North Carolina at Greensboro and the University of North Carolina at Chapel Hill approved the study (\#13-0311). This was a crosssectional study with data collection at 2 time points. The first was an in-person interview followed by a second telephone interview approximately 2 weeks later.

\section{Sample}

Based on a priori sample size estimation, a sample of 125 participants was estimated to be adequate for the multiple regression models, using a range of potential effect size estimates of 0.2 to 0.5 with the set standards of minimum coverage at $95 \%$, and estimate bias at less than $5 \%$, and target power $\geq 0$.8. However, a high recruitment rate allowed us to oversample within the study time line. Hence, a convenience sample of 198 pregnant women participating in WIC was recruited and interviewed during the 1-year study period. Women were recruited from the WIC clinic if they met the following selection criteria: (1) $\geq 18$ years of age, (2) 13 to 27 weeks pregnant (second trimester), and (3) able to speak either English or Spanish. Upon confirming the interest in study participation, women were asked to provide written consent for a first inperson interview and a second telephone interview.

\section{Measures}

The first interview that was conducted at the WIC clinic involved a collection of information on sociodemographics such as race/ethnicity, age, and income levels. Specifically, participants were grouped into the following racial/ethnic categories: (1) African American, (2) Hispanic, (3) non-Hispanic white, and (4) others, based on their self-report. The USDA 18item Food Security Survey Module was also used to assess food security status of the study population. ${ }^{20}$

For eating behaviors, the interview included questions on the frequency of meal skipping (for breakfast, lunch, and dinner) and the frequency of eating at fast food or other restaurants in a typical week. Participants who reported eating a particular meal $<5$ times a week were categorized as skipping that meal. The responses for frequency of eating at fast-food restaurants/ other restaurants per week were grouped into the following 3 conventional categories for the analyses: $\geq 5$ times/week, 2 to 4 times/week, and $\leq 1$ time/week.

For the assessment of diet quality, two 24-hour diet recalls were conducted, one of which was carried out during the first in-person interview. Following the end of the first in-person interview, the date and time for the second telephone interview was scheduled to conduct the second 24-hour dietary recall. The second telephone interview was scheduled to collect a combination of 1 weekday and 1 weekend day recall. Both the 24-hour recalls were conducted using the multiple-pass, paperpencil method, which is a validated 5-step approach developed by the USDA for collecting dietary recalls as accurately as possible. ${ }^{21}$ Participants were asked to recall specific types and quantities of foods they consumed over a 24-hour period, from midnight to midnight on the previous day. During the first inperson recall, participants were provided with food models, measuring spoons, glasses, and bowls to use as aids in recalling accurate estimations of the quantities of foods consumed. While for the second telephone recall, participants were asked to reference a "food amounts booklet" containing diagrams of concentric circles and square inch grids, as well as images of different sized serving utensils. ${ }^{22}$ The food amount booklet was distributed to the participants at the end of the first interview.

Of the total 198 women who participated in the in-person interview, 168 (85\%) completed the telephone recall. Although 
the aim was to collect 1 weekday and 1 weekend day recall for each participant, if they were not available for a specific day, priority was given to collect 2 recalls regardless of the day. Of the 168 women that completed both recalls, $68 \%$ completed 1 weekend and 1 weekday recall. Participants received a grocery store gift card to compensate for their time.

\section{Analysis}

The 24-hour recall data were analyzed using the 2013 Nutrition Data System for Research (NDSR) software (University of Minnesota, Nutrition Coordinating Center, Minneapolis, Minnesota). For a quality check, all dietary recalls for interviews 1 and 2 were reviewed to assess completeness and verification of portion sizes and amounts reported in original recall records. Discrepancies between recalled foods and beverages and available NDSR database options were resolved using the software manual's general data entry rules. For the analyses, the data from two 24-hour recalls were averaged, while data for participants with one 24-hour recall were left as is to calculate Healthy Eating Index-2010 (HEI-2010) score for each participant. The HEI2010 is a measure of an individual's diet quality in comparison to the recommendations of the national 2010 Dietary Guidelines for Americans (DGA) and consists of 12 component scores. ${ }^{23}$ Of the 12 components, 9 are adequacy components where intake is positively related to scores: total fruit, whole fruit, total protein foods, total vegetables, greens and beans, whole grains, dairy, seafood and plant proteins, and fatty acids. The remaining 3 components are referred to as moderation groups and are reverse-scored so that intake is negatively related to scores: sodium, refined grains, and empty calories. The total HEI2010 score, or the summation of the 12 component scores, ranges from 0 to 100 , with higher scores indicating a closer compliance with the 2010 DGA. A procedure of converting 2013 NDSR food group output files into HEI-2010 score was developed for this study using the guidelines available on the University of Minnesota Nutrition Coordinating Center website and the HEI-2010 methodology article by Guenther et al. ${ }^{24}$

All data for sociodemographics, eating behaviors, and HEI2010 total and component scores were analyzed using SPSS version 22.0 (IBM Corporation, Somers, New York). Descriptive statistics and frequencies were carried out for HEI-2010 scores, sociodemographics, food security status, and other variables. For the categories of race/ethnicity, as the size of the "Others" category was small, it was merged with the Hispanic group after confirming all the analyses results did not change significantly by merging these 2 groups. The bivariate 1-way analysis of variance (ANOVA) test was performed to estimate the mean difference in HEI-2010 score by race/ethnicity, other sociodemographic characteristics, and food security status. Ultimately, multivariate analysis was carried out to estimate differences in total HEI-2010 and its component scores by race/ ethnicity, after controlling for significant sociodemographic characteristics found in the bivariate analyses. For the eating behaviors of frequency of fast food consumption and meal skipping, a $\chi^{2}$ test was employed to analyze differences by race/ethnicity. The results were considered statistically significant at a $P$-value of $\leq .05$.

\section{Results}

About half (51\%) of the study participants were African American. The participants' average age was 26 years and for a majority, this pregnancy was unplanned (68\%). On average, participants' monthly household income was $\$ 1125$ $( \pm \$ 937)$. In terms of food security status, $43 \%$ of the participants experienced food insecurity.

\section{Healthy Eating Index-2010 Scores}

Descriptive statistics for total and component HEI-2010 scores are presented in Table 1. The average total HEI-2010 score for the entire sample was 56, ranging from a minimum of 21 to a maximum of 95 . Among the highly promoted or adequacy groups, the scores for whole grains, seafood and plant protein, and green and beans were lowest, with participants meeting recommendations only in the range of $33 \%$ to $40 \%$ of the maximum score. As shown in Table 1, the total protein group was scored the highest, and the average score for this component reflected $90 \%$ of the maximum score. For fruits and vegetables, the average percent scores of meeting the recommendation were $62 \%$ and $54 \%$, respectively (Table 1). Among the 3 moderation groups, the score for sodium was the lowest in reference to the standard of $\leq 1.1 \mathrm{~g} / 1000 \mathrm{kcal}$ for a maximum sodium score.

\section{Differences in HEI-20IO Scores by Sociodemographic Characteristics}

As shown in Table 2, a significant difference in the mean total HEI-2010 scores was seen by race/ethnicity, with African American women scoring lowest $\left(F_{2,195}=6.503, P=.002\right)$. By other sociodemographic characteristics, as shown in Table 2 , women in the younger age-group (18-25 years) had a significantly lower average total HEI-2010 score $\left(F_{1,196}=11.60, P\right.$ $=001$ ). Additionally, women in the single, divorced, or separated group had a significantly lower mean total HEI-2010 score than women who reported being married or living together with their partner $\left(F_{1,196}=13.45, P<.001\right)$. There was no significant difference in mean total HEI-2010 score by food security status. And although previous research has noted that having no car, and not receiving Supplemental Nutrition Assistance Program (SNAP) benefits are associated with poorer diet quality among low-income population, we found no significant association between these characteristics and diet quality among our study population.

In addition to total HEI-2010 scores, differences in individual component scores were assessed by race/ethnicity categories using ANOVA (results not shown). It was found that of the 12 HEI-2010 components, the following 6 components were significantly different between racial/ethnic groups: whole fruit, whole grains, dairy, total protein foods, sodium, 
Table I. Description of HEI-20I0 Scores for Pregnant Women Participating in WIC. ${ }^{\text {a }}$

\begin{tabular}{|c|c|c|c|c|c|c|}
\hline HEI-20IO & Maximum Score ${ }^{b}$ & Median & Min, Max & Mean (SD) & $95 \% \mathrm{Cl}$ for Mean & $\%$ Standard $\mathrm{Met}^{\mathrm{c}}$ \\
\hline \multicolumn{7}{|l|}{ Adequacy components } \\
\hline Whole fruit & 5 & 2.87 & $0.00,5.00$ & $2.77(2.08)$ & $2.48-3.06$ & 55 \\
\hline Whole grains & 10 & 2.89 & $0.00,10.00$ & $3.96(3.75)$ & $3.43-4.48$ & 40 \\
\hline Dairy & 10 & 5.14 & $0.00,10.00$ & $5.39(2.98)$ & $4.97-5.81$ & 54 \\
\hline Total vegetables & 5 & 2.48 & $0.00,5.00$ & $2.69(1.42)$ & $2.49-2.89$ & 54 \\
\hline Greens and beans & 5 & 0.39 & $0.00,5.00$ & $1.67(2.05)$ & $1.38-1.96$ & 33 \\
\hline Fatty acids & 10 & 4.36 & $0.00,10.00$ & $4.70(3.31)$ & $4.24-5.16$ & 47 \\
\hline \multicolumn{7}{|l|}{ Moderation components } \\
\hline Sodium & 10 & 2.47 & $0.00,10.00$ & $3.52(3.39)$ & $3.05-4.00$ & 35 \\
\hline Refined grains & 10 & 5.69 & $0.00,10.00$ & $5.49(3.55)$ & 4.99-5.99 & 55 \\
\hline
\end{tabular}

Abbreviations: $\mathrm{Cl}$, confidence interval; HEI, Healthy Eating Index; SD, standard deviation; WIC, Women, Infants, and Children.

${ }^{a} \mathrm{n}=198$.

${ }^{\mathrm{b}}$ The number indicates the highest possible score for total and each component of HEl-20IO.

${ }^{\mathrm{c}}$ The extent to which the total and component mean scores met the maximum score requirement.

and empty calories. Hence, as a next step, multivariate linear regression was conducted to estimate differences in total HEI2010 scores and these 6 components by race/ethnicity, after controlling for significant sociodemographics. As indicated in Table 3, of the promoted components, it was found that mean whole grains and dairy component scores were significantly lower for African American women compared to nonHispanic white women, after adjusting for age, education, planned pregnancy, and marital status. In contrast, African American women consumed more protein-based foods. Among the moderation components, African American women consumed higher amounts of sodium, and as a result, scored $\approx 2$ points lower for exceeding the daily sodium requirement compared to the reference group of non-Hispanic white pregnant women.

Among Hispanic women, the predicted mean intake scores of total fruits, dairy, total protein foods, and empty calories were significantly different compared to non-Hispanic white women. Hispanic pregnant women in this study had a greater intake of fruits, and as shown in Table 3, the predicted mean total fruit score was a point higher among Hispanic women compared to non-Hispanic women. Additionally, intake of empty calories was also significantly lower for Hispanic women, earning a predicted mean score $\approx 1.6$ points higher than the major racial/ethnic group of non-Hispanic white women.

\section{Differences in Eating Behaviors by Race/Ethnicity}

In meal skipping, breakfast skipping was the most common, with $40 \%$ reporting skipping it $\geq 2$ times/week, while $15 \%$ and $3 \%$ of the participants reported skipping lunch and dinner, respectively. About half of the women (48\%) reported eating at restaurant or consuming fast food consumption 3 or more times a week. In comparison, eating out or fast food consumption was significantly higher among African American women compared to 2 other racial/ethnic groups $(P=.025$, Figure 1$)$.

\section{Discussion}

The results indicate that the overall diet quality among our study population of pregnant women participating in WIC was suboptimal. The women in this study had a low consumption of dark green vegetables, beans/plant proteins, and whole grains, but the intake of sodium was very high. Similar to our study, a combination of high intake of sodium and low intake of whole grains was seen in the Bodnar et al study with 7511 nulliparous pregnant women recruited from 8 medical centers in the United States. Results of their study indicated that less than $5 \%$ of the participants met the recommended standards for whole grains, and about $3 \%$ met the recommendations for sodium intake. ${ }^{25}$ This is concerning because good nutrition is critical to promote normal growth and development during pregnancy. ${ }^{12,26-28}$ Optimal maternal nutrition requires an overall healthful dietary pattern and adequate intakes of critical micronutrients such as iron, folic acid, and calcium to support maternal health and fetal development during pregnancy. ${ }^{3}$

The results of our study indicate that the gaps in meeting the dietary guidelines are pronounced by maternal race/ethnicity, marital status, and age. Significantly lower total HEI-2010 scores were seen among younger women and single women, indicating that certain groups of low-income women are more vulnerable for poor diet quality and targeted prenatal nutrition programs and interventions are warranted. Being a single woman, having low social support, and bad partner relationships have all been previously identified as potential risk factors for excess gestational weight gain for low-income women. ${ }^{29}$ Furthermore, our results demonstrate that African 
Table 2. Differences in Total HEI-20I0 Score by Sociodemographics Among WIC Pregnant Women. ${ }^{\text {a }}$

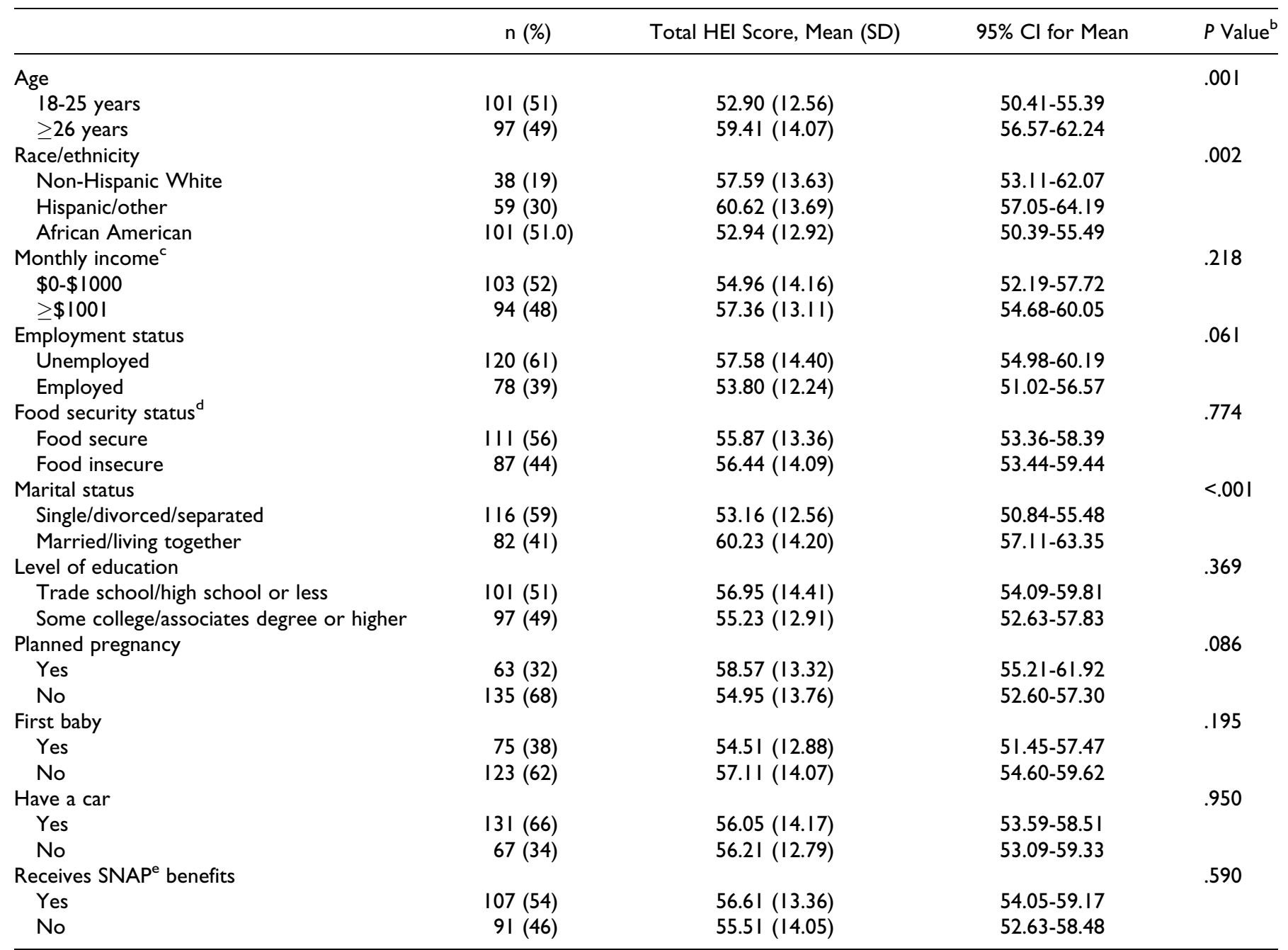

Abbreviations: $\mathrm{Cl}$, confidence interval; HEI, Healthy Eating Index; SD, standard deviation; SNAP, Supplemental Nutrition Assistance Program; WIC, The Special Supplemental Nutrition Program for Women, Infants, and Children.

$a_{n}=198$.

${ }^{\mathrm{b}}$ Results from I-way analysis of variance tests with independent variables and total HEl-20I0 score.

${ }^{c} n=197$, one person did not answer this question.

${ }^{d}$ Food secure denotes fully and marginally food secure women, food insecure denotes low and very low food secure women.

eThe Supplemental Nutrition Assistance Program.

American women were at the highest risk of experiencing poor dietary habits during pregnancy. This indicates that early-life nutrition disparities exist among African Americans, and at least in part might explain the higher rates of excess gestational weight gain and related poor birth outcomes of large for gestational age infants, greater infant adiposity, and increased cesarean and preterm delivery in this racial/ethnic group. ${ }^{12,30-32} \mathrm{~A}$ qualitative study of African American pregnant women investigating barriers to healthy eating found out that the expensive cost of healthy eating, lack of access to healthy foods, taste preferences, and cravings affected their ability to maintain healthy dietary habits. ${ }^{33}$

In our study, many women reported aberrant meal pattern of skipping breakfast frequently. This finding is notable as consuming a regular breakfast has been associated with better diet quality and a lower consumption of energy-dense foods later in the day. ${ }^{34-36}$ Additionally, regular consumption of fast-foods was significantly higher among African American women, which in turn might explain the high intake of sodium and animal protein and low intake of fiber among this group of population. ${ }^{37,38}$

In our study, we did not find any differences in diet quality between food secure and insecure participants. However, strong evidence exists indicating that food-insecure households are likely to have reduced physical and economic access to nutrient-dense foods such as fruits and vegetables. ${ }^{39,40}$ By specifically reaching a homogenous group of low-income women, it is possible that difference in diet quality by food security status was masked. Furthermore, it is possible that the development of pregnancy changes the dynamics of intrahousehold 
Table 3. Differences in Health Eating Index-20I0 Scores by Race/ Ethnicity Among WIC Pregnant Women.,

\begin{tabular}{|c|c|c|c|c|}
\hline \multirow{2}{*}{$\begin{array}{l}\text { Healthy Eating } \\
\text { Index-2010 }\end{array}$} & \multicolumn{2}{|c|}{ African American } & \multicolumn{2}{|c|}{ Hispanic/Other } \\
\hline & $\beta$ & $95 \% \mathrm{Cl}$ & $\beta$ & $95 \% \mathrm{Cl}$ \\
\hline Total score & -3.01 & -7.97 to 1.94 & 1.18 & -4.20 to 6.56 \\
\hline \multicolumn{5}{|c|}{ Adequacy components } \\
\hline Total fruit & 0.21 & -0.52 to 0.96 & $0.98^{c}$ & 0.17 to 1.79 \\
\hline Whole grains & $-I .7 I^{c}$ & -3.10 to -0.32 & -0.25 & -1.76 to 1.25 \\
\hline Dairy & $-1.42^{c}$ & -2.51 to -0.33 & $-1.0 I^{d}$ & -2.19 to 0.17 \\
\hline $\begin{array}{l}\text { Total protein } \\
\text { foods }\end{array}$ & $0.64^{c}$ & 0.23 to 1.04 & $0.39^{d}$ & -0.04 to 0.83 \\
\hline \multicolumn{5}{|c|}{ Moderation components } \\
\hline Sodium & $-1.70^{c}$ & -3.00 to $-0.4 I$ & -0.48 & -1.89 to 0.92 \\
\hline Empty calories & 0.33 & -1.05 to 1.72 & $1.57^{c}$ & 0.06 to 3.09 \\
\hline
\end{tabular}

Abbreviations: $\mathrm{Cl}$, confidence interval; WIC, The Special Supplemental Nutrition Program for Women, Infants, and Children.

${ }^{a} \mathrm{n}=198$.

bultiple linear regression; adjusted for education, age, planned versus unplanned pregnancy, marital status.

For comparison by ethnic groups, dummy variables were created, and nonHispanic white group was used as a reference category.

${ }^{\mathrm{c}} P \leq .05$.

${ }^{\mathrm{d}} \mathrm{P} \leq .10$.

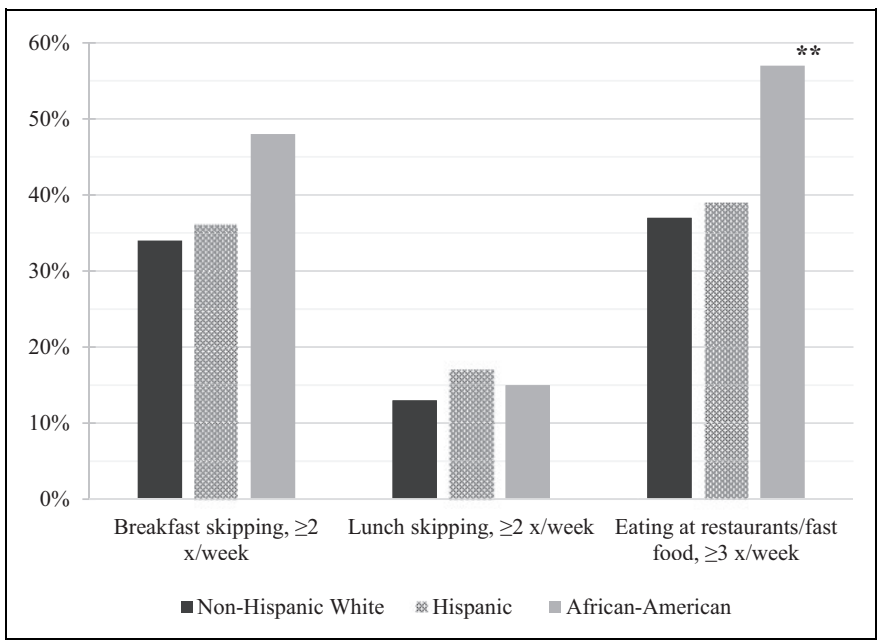

Figure I. Differences in the frequency of eating behaviors by race/ ethnicity among Women, Infants, and Children (WIC) pregnant women $(n=198)$.

food allocation, such that the dietary intake of the pregnant woman remains protected and stable. Previous research has shown that a "buffering effect" occurs and vulnerable groups such as children are given a high priority in household food distribution. ${ }^{41}$ Future investigation into the home food environment and family dynamics might provide insight into how and to what extent pregnant women are protected from food insecurity.

There are several limitations to this study. Most notably, this study was conducted with a convenience sample of low-income pregnant women at a singular WIC clinic, limiting the generalizability of the results. However, the racial/ethnic distribution in our study represents the national trend. The results of this

\section{SO WHAT?}

\section{What is already known on this topic?}

Low-income and minority women in the United States are at an increased risk of excess weight gain during pregnancy, and this is a critical factor in causing earlylife nutrition and health disparities.

\section{What does this article add?}

Results of this study provide quantitative estimate on the extent to which the Dietary Guidelines are met by lowincome women during pregnancy. Specifically, the study demonstrates nutrition disparities occur among lowincome women during pregnancy, with African American women being the most vulnerable group for poor diet quality.

\section{What are the implications for health promotion practice or research?}

Results of this study indicate that nutrition education programs and interventions designed to improve the diet quality of racial/ethnic minority women are warranted. Interventions promoting regular meal patterns and nutrient-dense food choices may be effective in improving diet quality and addressing health disparities. The excessive intake of sodium among our study participants underscores the importance of implementing policies and regulations to help decrease sodium in processed food and promote awareness on regulating sodium intake during pregnancy.

study may not be applicable to eligible women not participating in the WIC program. Furthermore, the component score for "empty calories" typically incorporates solid fat, added sugar, and alcohol intake. For our participants, due to pregnancy, alcohol intake was not common. We therefore speculate that the score for empty calories would have been lower if it considered only added sugars and solids fats.

\section{Authors' Note}

A.M.H. organized the data, converted 24-h diet recall information for nutrient and food group analyses, wrote initial draft of the manuscript. D.L.N. participated in the development of the study materials, recruitment, collection of data. A.A. served as the consultant in finalizing data collection materials. J.M.D. designed the study and its goals and objectives and procured funding. In her role as a principal investigator, she supervised the study activities, IRB documentation, community outreach, and conducted final data analyses and manuscript revisions.

\section{Declaration of Conflicting Interests}

The author(s) declared no potential conflicts of interest with respect to the research, authorship, and/or publication of this article. 


\section{Funding}

The author(s) disclosed receipt of the following financial support for the research, authorship, and/or publication of this article: The study was supported by the National Center for Advancing Translational Sciences (NCATS), National Institutes of Health, through Grant Award Number UL1TR002489. The content is solely the responsibility of the authors and does not necessarily represent the official views of the NIH.

\section{ORCID iD}

Alla M. Hill (D) https://orcid.org/0000-0002-1550-5293

\section{References}

1. Rifas-Shiman SL, Rich-Edwards JW, Kleinman KP, Oken E, Gillman MW. Dietary quality during pregnancy varies by maternal characteristics in project viva: a US cohort. $J$ Am Diet Assoc. 2009;109(6):1004-1011. doi:10.1016/j.jada.2009.03.001.

2. Abu-Saad K, Fraser D. Maternal nutrition and birth outcomes. Epidemiol Rev. 2010;32:5-25. doi:10.1093/epirev/mxq001.

3. Campbell CG, Procter SB. Position of the academy of nutrition and dietetics: nutrition and lifestyle for a healthy pregnancy outcome. J Acad Nutr Diet. 2014;114(7):1099-1103. doi:10.1016/j. jand.2014.05.005.

4. Fowles ER, Gabrielson M. First trimester predictors of diet and birth outcomes in low-income pregnant women. $J$ Community Health Nurs. 2005;22(2):117-130. http://www.jstor.org/stable/3 427971.

5. Kind KL, Moore VM, Davies MJ. Diet around conception and during pregnancy-effects on fetal and neonatal outcomes. Reprod Biomed Online. 2006;12(5):532-541. doi:10.1016/ S1472-6483(10)61178-9.

6. Brantsæter AL, Haugen M, Samuelsen SO, et al. A dietary pattern characterized by high intake of vegetables, fruits, and vegetable oils is associated with reduced risk of preeclampsia in nulliparous pregnant Norwegian women. $J$ Nutr. 2009;139(6):1162-1168. doi:10.3945/jn.109.104968.

7. Frederick IO, Williams MA, Dashow E, Kestin M, Zhang C, Leisenring WM. Dietary fiber, potassium, magnesium and calcium in relation to the risk of preeclampsia. J Reprod Med. 2005;50(5):332-344.

8. Qiu C, Coughlin KB, Frederick IO, Sorensen TK, Williams MA. Dietary fiber intake in early pregnancy and risk of subsequent preeclampsia. Am J Hypertens. 2008;21(8):903-909. doi:10.1038/ajh.2008.209.

9. Oken E, Ning Y, Rifas-Shiman SL, Rich-Edwards JW, Olsen SF, Gillman MW. Diet during pregnancy and risk of preeclampsia or gestational hypertension. Ann Epidemiol. 2007;17(9):663-668. doi:10.1016/j.annepidem.2007.03.003.

10. Xu H, Shatenstein B, Luo ZC, Wei S, Fraser W. Role of nutrition in the risk of preeclampsia. Nutr Rev. 2009;67(11):639-657. doi:10.1111/j.1753-4887.2009.00249.x.

11. Waterland RA, Jirtle RL. Early nutrition, epigenetic changes at transposons and imprinted genes, and enhanced susceptibility to adult chronic diseases. Nutrition. 2004;20:63-68. doi:10.1016/j. nut.2003.09.011.
12. Guelinckx I, Devlieger R, Beckers K, Vansant G. Maternal obesity: pregnancy complications, gestational weight gain and nutrition. Obes Rev. 2008;9(2):140-150. doi:10.1111/j.1467-789X. 2007.00464.x.

13. Hanley B, Dijane J, Fewtrell M, et al. Metabolic imprinting, programming and epigenetics - a review of present priorities and future opportunities. Br J Nutr. 2010;104(suppl 1):S1-25. doi:10.1017/S0007114510003338.

14. Gamba R, Leung CW, Guendelman S, Lahiff M, Laraia BA. Household food insecurity is not associated with overall diet quality among pregnant women in NHANES 1999-2008. Matern Child Health J. 2016;20(11):2348-2356. doi:10.1007/s10995016-2058-1.

15. Siega-Riz AM, Bodnar LM, Savitz DA. What are pregnant women eating? Nutrient and food group differences by race. Am J Obstet Gynecol.2002;186(3):480-486. doi:10.1067/mob.2002.121078.

16. Rosenkranz RR, Dzewaltowski DA. Model of the home food environment pertaining to childhood obesity. Nutr Rev. 2008; 66(3):123-140. doi:10.1111/j.1753-4887.2008.00017.x.

17. Anderson B, Rafferty AP, Lyon-Callo S, Fussman C, Imes G. Fast-food consumption and obesity among Michigan adults. Prev Chronic Dis. 2011;8(4):A71.

18. Hunger and Obesity: Understanding a Food Insecurity Paradigm: Workshop Summary. Washington, DC: Institute of Medicine; 2011.

19. USDA - FNS. WIC Eligibility and Coverage Rates. www.fns.us da.gove/WIC. Accessed October 15, 2019.

20. U.S. Household food Security Survey Module: Three-Stage Design, with Screeners. Washington, DC: US Department of Agriculture Economic Research Service; 2012.

21. Conway JM, Ingwersen LA, Vinyard BT, Moshfegh AJ. Effectiveness of the USDA 5-step multiple-pass method to assess food intake in obese and non-obese women. Am J Clin Nutr. 2003; 77(5):1171-1178.

22. Valencia A, Stevens M.Food amounts booklet for the participant. Nutrition Coordinating Center. 2007. http://www.ncc.umn.edu/ products/support/food-amount-reporting-booklets/. Accessed June, 2014.

23. Guenther PM, Kirkpatrick SI, Reedy J, et al. The Healthy Eating Index-2010 is a valid and reliable measure of diet quality according to the 2010 dietary guidelines for Americans. J Nutr. 2014; 144(3):399-407. doi:10.3945/jn.113.183079.

24. Guenther PM, Casavale KO, Reedy J, et al. Update of the Healthy Eating Index: HEI-2010. J Acad Nutr Diet. 2013;113(4):569-580. doi:10.1016/j.jand.2012.12.016.

25. Bodnar LM, Simhan HN, Parker CB, et al. Racial or ethnic and socioeconomic inequalities in adherence to national dietary guidance in a large cohort of US pregnant women. J Acad Nutr Diet. 2017;117(6):867-877.e3. doi:10.1016/j.jand.2017.01.016.

26. Weight Gain During Pregnancy: Reexamining the Guidelines. Washington, DC: Institute of Medicine and National Research Council; 2009.

27. Nehring I, Schmoll S, Beyerlein A, Hauner H, Von Kries R. Gestational weight gain and long-term postpartum weight retention: a meta-analysis. Am J Clin Nutr. 2011;94(5):1225-1231. doi:10.3945/ajen.111.015289. 
28. Hernandez DC. Gestational weight gain as a predictor of longitudinal body mass index transitions among socioeconomically disadvantaged women. J Womens Health. 2012;21(10): 1082-1090. doi:10.1089/jwh.2011.2899.

29. Paul KH, Graham ML, Olson CM. The web of risk factors for excessive gestational weight gain in low income women. Matern Child Health J. 2013;17(2):344-351. doi:10.1007/s10995-012-0979-x.

30. Savitz DA, Stein CR, Siega-Riz AM, Herring AH. Gestational weight gain and birth outcome in relation to prepregnancy body mass index and ethnicity. Ann Epidemiol. 2011;21(2):78-85. doi: 10.1016/j.annepidem.2010.06.009.

31. Headen IE, Davis EM, Mujahid MS, Abrams B. Racial-ethnic differences in pregnancy-related weight. Adv Nutr. 2012;3: 83-94. doi:10.3945/an.111.000984.83.

32. Bryant AS, Worjoloh A, Caughey AB, Washington AE. Racial/ ethnic disparities in obstetric outcomes and care: prevalence and determinants. Am J Obstet Gynecol. 2010;202(4):335-343. doi:10.1016/j.ajog.2009.10.864.

33. Whitaker KM, Wilcox S, Liu J, Blair SN, Pate RR. African American and White women's perceptions of weight gain, physical activity, and nutrition during pregnancy. Midwifery. 2016;34: 211-220. doi:10.1016/j.midw.2015.11.005.

34. Kant AK, Andon MB, Angelopoulos TJ, Rippe JM. Association of breakfast energy density with diet quality and body mass index in American adults: National Health and Nutrition Examination Surveys, 1999-2004. Am J Clin Nutr. 2008;88:1396-1404. doi:10.3945/ajen.2008.26171.1.
35. Widaman AM, Witbracht MG, Forester SM, Laugero KD, Keim NL. Chronic stress is associated with indicators of diet quality in habitual breakfast skippers. J Acad Nutr Diet. 2016;116(11): 1776-1784. doi:10.1016/j.jand.2016.03.016.

36. O'Neil CE, Nicklas TA, Fulgoni VL. Nutrient intake, diet quality, and weight/adiposity parameters in breakfast patterns compared with no breakfast in adults: National Health and Nutrition Examination Survey 2001-2008. J Acad Nutr Diet. 2014;114(12): S27-S43. doi:10.1016/j.jand.2014.08.021.

37. Prentice AM, Jebb SA. Fast foods, energy density and obesity: a possible mechanistic link. Obes Rev. 2003;4(4):187-194. doi:10.1046/j.1467-789X.2003.00117.x.

38. Darmon N, Drewnowski A. Contribution of food prices and diet cost to socioeconomic disparities in diet quality and health: a systematic review and analysis. Nutr Rev. 2015;73(10):643-660. doi:10.1093/nutrit/nuv027.

39. Larson NI, Story MT, Nelson MC. Neighborhood environments. Disparities in access to healthy foods in the U.S. Am J Prev Med. 2009;36(1):74-81. e10. doi:10.1016/j.amepre.2008.09.025.

40. Baker EA, Schootman M, Barnidge E, Kelly C. The role of race and poverty in access to foods that enable individuals to adhere to dietary guidelines. Prev Chronic Dis. 2006; 3(3):A76. doi:A76 [pii].

41. McIntyre L, Glanville TN, Raine KD, Dayle JB, Anderson B, Battaglia N. Do low-income lone mothers compromise their nutrition to feed their children? Can Med Assoc J. 2003;168(6): 686-691. 